\title{
Condition Assessment of Electrical Connections Utilizing Infrared Thermography
}

\author{
G. B. McIntosh
}

Snell Infrared Canada, 556 Edward Ave., Suite 58, Richmond Hill, ON, Canada,gmcintosh@thesnellgroup.com

\begin{abstract}
Thermography is well established as the best non-contact method for detecting defective electrical connections. But determining the severity of the defect is often based upon temperature, or temperature rise above normal operating temperature. Surface temperature alone, however, is often an unreliable indicator of condition or severity. This paper will discuss various considerations for assessing the condition of an electrical connection exhibiting a thermal anomaly. This discussion will be limited to electrical connections and not electrical devices such as circuit breakers, fuses, capacitors, etc., but will apply to connections attached to these devices, albeit with some caveats which will be discussed.
\end{abstract}

\section{Introduction}

The established method for identifying a problem with electrical connections is observing it via line of sight with

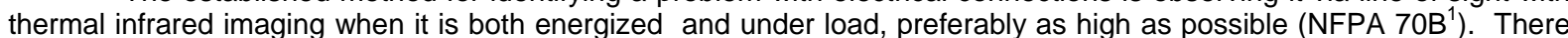
is, however a great misunderstanding about how to establish the condition of the connection once a thermal anomaly has been found. Historical methods have applied the use of temperature, or temperature rise, established with the infrared camera, as an indicator of the defect severity. Infrared thermography, however, only identifies surface temperature rather than internal interface temperature, and therefore surface temperature taken alone is an unreliable indicator of the fault severity ${ }^{2}$. Some severity assessment methods have combined the use of component specific temperature regimes, while others have used load and environment correction factors. The closest we have to absolute limits are the maximum temperature ratings of the wire, connector, or device attached to the connector. This limit is typically $60^{\circ} \mathrm{C}$, $75^{\circ} \mathrm{C}$, or $90^{\circ} \mathrm{C}$. When an NMD -75 wire $\left(75^{\circ} \mathrm{C}\right.$ limit) is attached to a Cu9Al $\left(90^{\circ} \mathrm{C}\right.$ limit) and an unrated $\left(60^{\circ} \mathrm{C}\right.$ default) device rated then the connection limit is $60^{\circ} \mathrm{C}^{3,4}$. But when a connector fault produces a surface temperature of less than $60^{\circ} \mathrm{C}$ (e.g. because of load or low ambient temperature) there is a variety of conflicting opinions as to what severity (action) should be taken. The reality is there cannot be one single set of temperature regimes other than the limits stated above which can predict how bad a particular connection is, or how close to failure it may be.

The core value of thermography is being able to identify that a thermal anomaly exists. While thermography can identify a connection problem through thermal pattern analysis, the connector surface temperature can be very a poor indicator of the nature of the problem or its severity. As illustrated in Figure 1, the three phase connections are equally loaded at less than $10 \%$ of the rated capacity of the circuit and the defective connection, shown on the right phase, is "only" exhibiting less than a $10^{\circ} \mathrm{C}$ rise above the normal operating temperature of the left and center phases. Yet after analysis this potential fault can be categorized as a functional failure, which could become a complete catastrophic failure at higher circuit loading, since fault power increases as the square of the current. Further, in the or event of a fault current, this supposedly "minor defect" could fail dramatically as an arc flash explosion with potential for loss of life, large capital loss and interruption of service. Ironically, reducing the risk of electrical fire and explosion is one of the key reasons that thermography is utilized. ${ }^{1}$

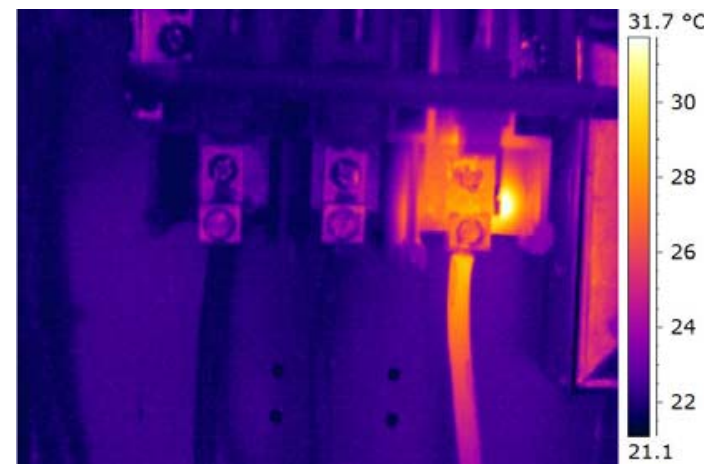

Figure 1. A connector exhibiting a thermal anomaly 
To establish the true "condition" of a defective electrical connection the fundamental issue of root cause as to why the connector or component is running warm should be known. A connection should not run warmer than the conductor attached to it. If it is, then it is not performing as it was intended and therefore has "functionally" failed. Whether this anomaly will ever catastrophically fail, is immaterial, since we can never know or predict the stressors that in the future may be placed on this connection which may cause it to "catastrophically" fail.

This paper only deals with stand-alone electrical connections. It does not deal with thermal devices (e.g. fuses, circuit breakers, overloads, contactors, capacitors) and for the most part does not deal with the first connection immediately attached to such device, although it does cover the second and subsequent connections adjacent to the device. (In other words it does not cover a fuse clip but includes the wire connection to the clip itself). For these types of connections please refer to the discussion at the end of this paper "primary connections attached to thermal devices".

\section{Types of Failure}

As defined by Nolan and Heap in their what is now classical reference document ${ }^{5}$ for reliability there are three types or classifications of failure: potential; functional; and complete. For the purposes of evaluating electrical connections we will add a 4th classification: catastrophic which, in reality, is a type of complete failure. This is an important distinction because many maintenance managers typically only consider two possibilities when evaluating thermographic findings of electrical components: potential (it may never happen) and catastrophic (it open circuits). If however we are to apply sound principles of reliability centered maintenance to electrical systems, and in particular electrical connections, then we must have multiple definitions of failure as follows:

2.1. Potential failure: "A detectable symptom or warning sign that a functional failure is in the process of occurring". In this definition Nolan and Heap ${ }^{5}$ note that potential failures are often detected by maintenance personnel. In the case of electrical connections every connection warmer than the conductor attached to it can be considered a potential failure. And the best way to detect a potential connection is by detecting a thermal anomaly on that connection with thermography.

2.2 Functional failure: "The inability of any asset to fulfill a function to a standard of performance which is acceptable to the user" (Moubray ${ }^{6}$ ). Nolan and Heap note that functional failures are often detected by operational personnel but in the case of redundant systems may go undetected. For electrical systems this means that a connection will fail to meet one or more of its functional design criteria. Unfortunately the nature of most electrical connections is such that it is hard to know if a functional failure has occurred until a complete catastrophic failure exists. Sometimes connector discoloration, melting insulation, noticeable arcing and/or RF interference may be indications that a functional failure has occurred.

2.3 Failure: "The termination of the ability of an item to perform a required function". (ISO $14224^{7}$ ) In the case of an electrical connection this typically means that melting has occurred within the connection. This melting produces one of two results: a catastrophic failure; or a re-welding of the component. In the case of re-welding on a interruptible connection (e.g.: the hinge or jaws of a switch) the connection continues to operate but when called upon to function, the switch will not be able to open. Also called a "complete failure" 5

2.4. Catastrophic (or critical) failure: "A form of complete failure which results in immediate interruption of operation or use" ${ }^{2}$. It may involve capital equipment loss, loss of life or loss of profit. In the case of an electrical connection it means at best an open circuit and interruption of service or at worst an arc-flash explosion and interruption of service, loss of capital, collateral damage and perhaps injury or loss of life. ${ }^{8}$

\section{The Challenge: Potential, Functional or Complete Failure?}

Many maintenance managers expect that a thermographer (contractor or employee) will tell them whether or not a thermal anomaly is a problem that needs to be fixed or not, and the time frame for doing so. Without more information, however and to some extent a crystal ball, this is an impossible demand of any thermographer. Every thermal anomaly is definitely a potential failure and may in fact be a functional or even in some cases a complete failure. And it is quite possible that an external unexpected stressor can rapidly turn a functional failure into a catastrophic failure. Differentiating between a potential and functional failure often requires analysis that may not always be possible on live energized electrical equipment.

The challenge therefore is clear: maintenance managers do not typically have the resources to repair every "potential" failure, yet from a reliability centered maintenance perspective they must be prepared to repair any "functional" failure. The thermographer must be able to differentiate between the two, and that is not always possible without additional testing and analysis tools. 


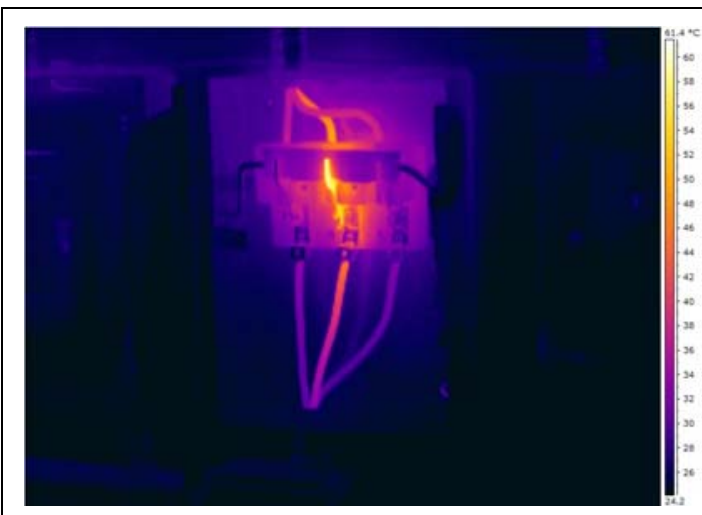

\section{Figure 2a: An example of potential failure}

The jaws on this disconnect are running warm but without further analysis the only determination at this point is that it is a potential failure. With sufficient fault power the failure mode of this component could be such that contact welding takes place resulting in complete but not catastrophic failure. This could turn into a catastrophic failure, however, if it does weld itself and an operator tries to operate (open-circuit) the device to isolate the equipment, the disconnect may fail causing an arc-flash explosion and possible severe injury or death.

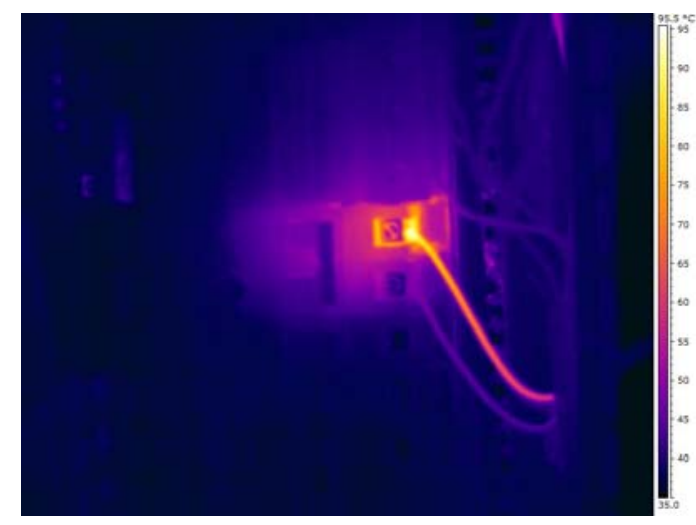

\section{Figure 2b: An example of functional failure}

This connector is running at a temperature exceeding the rating of the wire insulation attached to it (90 C) Running above this temperature may cause annealing of the metal and may be high enough to reach melting voltage on the contacts. At this point complete failure is unpredictable but likely imminent before the next routine (often yearly) inspection. Failure likely will be catastrophic resulting in interruption of service at the very least.

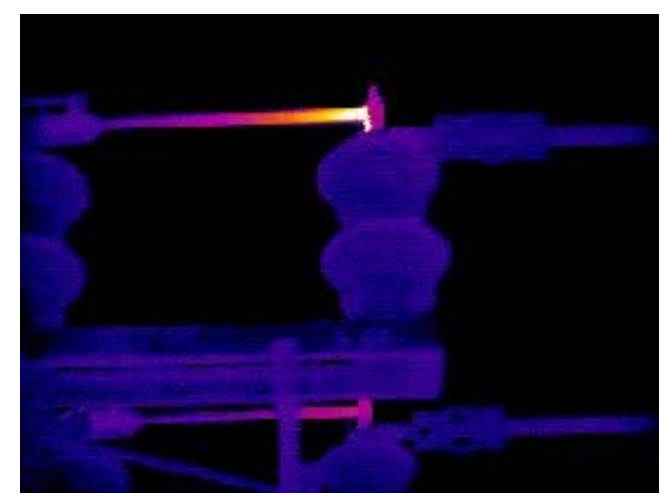

Figure 2c:An example of complete failure

The function of an outdoor switch is to isolate the circuit for maintenance. A thermal anomaly such as this may never cause a catastrophic failure (open circuit) but it has functionally failed if the melting voltage has been exceeded and welded the switch, making isolation of the circuit during a scheduled outage difficult or even impossible.

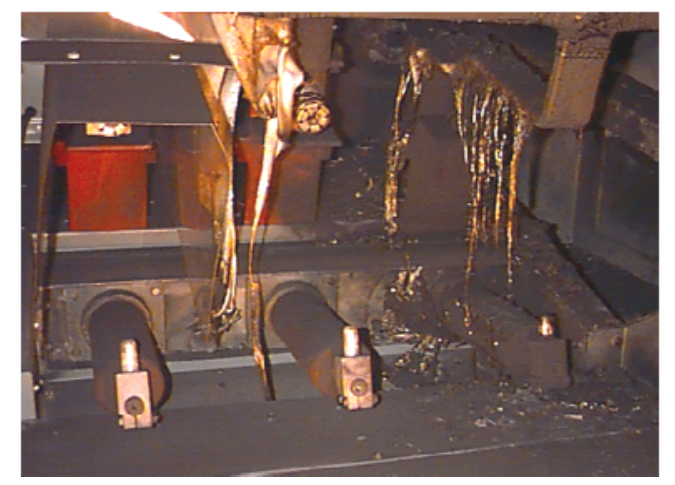

Figure 2d: An example of catastrophic (or critical) failure There are usually no thermal images of catastrophic failure because once it happens a circuit is no longer intact. Only visual pictures of the aftermath. Failure of high energy electrical connections typically results in significant damage to the conductor, adjacent equipment, and interruption of service. The explosive nature of an arc-flash explosion, caused by vaporizing metal, may cause fire, injury and loss of life. It is why thermography is identified as being necessary for all critical electrical distribution systems. Photo courtesy of Bermuda Electric and Light Company. 


\section{Establishing Functional Failure}

In order to establish whether functional failure has occurred one must understand the design (functional) criteria used for the connection. In simple terms the primary purpose of an electrical connection is to transport electrical current as effectively as the conductor (s) attached to it. There are however, three different conditions for this transport:

1) To transport the design maximum continuous electrical current as efficiently as the electrical conductor attached to it. This is typically defined by electrical code as $80 \%$ of the rating of the protective device (a fuse or breaker). A circuit protected by a 15 amp fuse should be capable of carrying 12 amps continuously.

2) To transport the design maximum short-term electrical current as efficiently as the conductor attached to it. This allows for temporary short term higher currents created by devices such as motors during start-up. Typically these in-rush currents could be up to 8 times greater than the rated full load current and last up to a few seconds. Special breakers or fuses are specifically designed to allow this short term current and so should the connectors and conductors in the circuit. This temporary high current condition may cause thermal warming in the connection for which it should be able to dissipate easily.

3) To transport the design instantaneous short circuit current as efficiently as the conductor attached to it. Short circuit currents can be thousands of times the rated continuous circuit capacity. This instantaneous, extremely high current condition may cause thermal warming in the connection for which it should be able to dissipate without causing thermal runaway. (thermal runaway is the condition whereby heat is generated faster in the component than it can be dissipated)

In addition for bolted and operable connections:

4) To maintain the three general criteria listed above, plus allow for the connected component removal, disassembly, replacement, or disconnection either on a routine or periodic basis. Examples are a fused disconnect, bolted connection, isolation switch, etc.

In all four situations the connector should suffer no undo damage, degradation or reduction in service life. Other design criteria for a connector may include allowance for such things as vibration, corrosion, load cycling, thermal cycling, dissimilar metals, operation, etc.

In general, this means that electrical connections should have at least the equivalent unit surface area as the conductor attached to it. (if this were true the connection should have the same unit resistance (power dissipation loss) as the conductor and therefore run at approximately the same temperature) In practice however, a design factor for connectors typically is applied such that that the connector has much greater unit surface area than the conductor attached to it. This allows "forgiveness" for the unexpected such as improper installation or connector degradation over time, resulting in decreased contact surface area. Theoretically, a properly installed and maintained electrical connector should have lower unit resistance and therefore less unit power loss (heat generation) than the conductor attached to it. For the purposes of electrical thermography we should expect that a connection currently meets its design criteria if, under load, it is not running any warmer than the conductor attached to it.

Theoretically, by design a connection under load and not attached to a thermal device (fuse, circuit breaker, overload, etc.,) should run no warmer than the conductors attached to it. In practice, however, as can be seen in Figure 2 , there may be a very slight uniform warming as the conductor approaches the connector as the free convective cooling decreases.

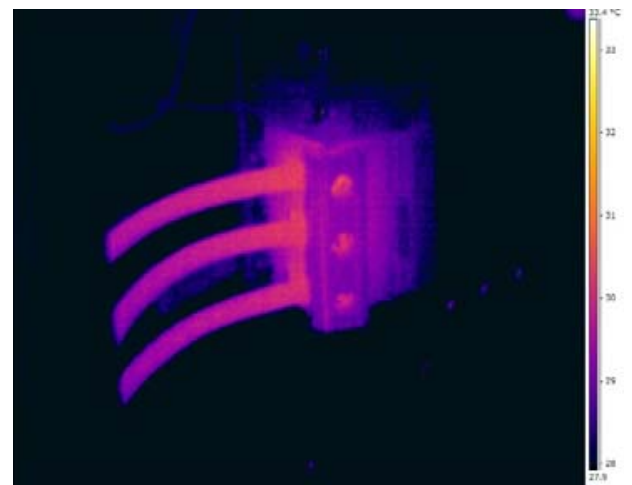

Figure 3. An example of a good connection under load. 


\section{Electrical Connection Functional Failure Root Cause}

Understanding the root cause of an electrical connection "functional failure" is essential to assessing the risk of catastrophic failure. Unfortunately an infrared camera is not a thermal x-ray - it only observes surface temperature patterns and not the actual internal temperature at the connection. The reality is that the temperature at the internal contact points (in a faulty connection under load) will be much higher than the external temperature of the surface. ${ }^{10}$

There are many possible reasons for a warm connector, the most obvious and misdiagnosed of which is a "loose" connection. This term alone is a misnomer and should properly be termed "under-torque". Even if a connector has the proper torque initially, the contact force in the electrical interfaces may decay due to poor connector design leading to overheating, ensuing metal creep/flow and ensuing further decrease in contact force. In addition to issues related to under-torque, a connection could be warped, dirty, corroded, or even over-torque to the point where it has been cold-worked with resultant stress cracking. While these may be symptoms causing the warm connection, they are not the "root cause" nor do they describe the potential failure mode. Connector failure root causes are shown in Table 1.

Table 1. Various Reasons for Connector Failure

\begin{tabular}{|l|l|}
\hline Improper design & Material compatibility \\
\hline Improper preparation and assembly & Improper torque \\
\hline Excessive vibration & Corrosive environment \\
\hline Excessive/thermal environment & Single event over-stress \\
\hline
\end{tabular}

\section{Why would a thermal anomaly get worse on its own?}

The easy answer to this is that something is occurring to continually reduce the contact surface area of the connection. The common reason for this is thought to be fretting corrosion which is the micro-movement of one surface against the other. Three potential sources for this movement are external vibration, harmonic vibration, and thermal cycling. A second cause for surface area reduction could be the presence of micro-arcing in the air gap between a warped or mal-fit connection where a slight air gap exists. A third cause for this surface area reduction is the presence of an external corrosive agent

The most troubling aspect of all the above factors is it is unlikely that without further testing, or perhaps even without disassembly, we may never be able to ascertain what is causing the lack of contact. It may be possible, in some instances to use an airborne ultrasonic device, to detect if there is internal arcing within the connection but this is just one possible internal phenomenon. In some instances it may be possible to measure a voltage drop across the connection in which case the impedance and proximity to melting voltage can be established. But in most cases this is not possible, safe, and/or practical.

\section{How to Deal with a Warm Connection}

\subsection{Perform a Risk Assessment}

The simple and easiest way to deal with every connector thermal anomaly (identified using NFPA 70b criteria) detected is to assume that it has functionally failed and should be investigated and repaired. In some cases such as a 9 am to $5 \mathrm{pm}$ operation, this can often be done without significant disruption or cost other than maintenance time and material. This will produce the highest reliability but perhaps at the greatest short term maintenance cost. When maintenance resources are limited, high reliability is not required, or in a 24/7/365 operation where an outage means production interruption, this approach is typically not practical and/or cost effective. In these cases, one has to consider the probability and consequence of an unexpected failure and make an appropriate risk-management decision.

In order to assess the probability of failure one must take the following into consideration:

- What is causing the lack of contact

- Is the anomaly going to get worse on its own

- How close is the anomaly to complete failure

- What are the potential likely stressors that will accelerate complete failure

- What are the potential but unlikely stressors that may cause rapid complete failure

The second part of any risk management assessment is the consequence of unexpected failure:

- What is the potential for personnel injury (arc flash explosion, fire)

- What is the potential for loss of service (loss of use, profit) 
- What is the potential for loss of capital (electrical components)

- What is the potential for consequent damage (damage to downstream equipment, environmental harm)

- What is the potential for collateral damage (fire and explosion damage)

\subsection{Failure Assessment}

There are 2 possible courses of action for assessment of proximity to "complete failure": the Wattage approximation method; and thermal modelling.

\subsubsection{The Wattage Approximation Method}

To employ this method we need direct line of sight to the anomaly and to know the actual current in the circuit at the time of inspection. Trained and experienced Level 2 thermographers should understand thermal pattern analysis and try to anticipate the source and extent of the problem. For analysis purposes it is important to consider the total surface area affected (heat affected zone), the material and mass of the device, the surrounding radiant and convective conditions, as well as the maximum and average temperature of the heat affected zone. Then they simply ask the question: "what is the my best guess as to what amount of wattage would create a thermal pattern on the surface of this size and temperature?" An order of magnitude wattage approximation is all that is required. When even an order of magnitude approximation is not possible, an estimate may be made based upon Stefan-Boltzmann radiation heat loss added to a convective heat loss approximation (e.g. obtained through Newton's or Langmuir's relationships). In these calculations the total area used is that of the heat affected zone and the surface temperature is the average of the heat affected zone obtained by thermography.

The next step is take the measured current flowing at the time and calculate the order of magnitude fault resistance by dividing the estimated wattage by the current squared. This will yield an order of magnitude resistance in Ohms of the connection. Multiply by 1000 to obtain the value in milli-Ohms or by an additional 1000 to obtain the value in micro-Ohms.

Once the micro-Ohm value has been established then apply the design current to calculate the potential voltage drop across the connection. If this value approaches the "melting voltage" value, there is a high probability for complete failure. Please refer to Table 2 for melting voltage values, Figure 4 for a worked example, and Reference [9] for further discussion on this topic.

Table 2. Softening and Melting Voltages for Some Metals ${ }^{9}$

\begin{tabular}{|l|c|c|}
\hline Material & Softening Voltage & Melting Voltage \\
\hline Al (Aluminium) & 0.1 & 0.3 \\
\hline $\mathrm{Cu}$ (Copper) & 0.12 & 0.43 \\
\hline $\mathrm{Ag}$ (Silver) & 0.09 & 0.37 \\
\hline $\mathrm{Au}$ (Gold) & 0.08 & 0.43 \\
\hline $\mathrm{Sn}$ (Tin) & 0.07 & 0.13 \\
\hline $\mathrm{Zn}$ (Zinc) & 0.1 & 0.17 \\
\hline $\mathrm{Ni}$ (Nickel) & 0.16 & 0.16 \\
\hline
\end{tabular}

The disadvantage of this method is that it cannot deal with design criteria (2) or (3) since these are transient events and the thermal capacitance of the material will come into play as to whether actual melting temperature is achieved. In addition high forced convection conditions, such as high wind, preclude the use of this technique.

\subsubsection{Thermal Modelling Method}

This method involves the use of a three dimensional computer aided design model of the component and the use of a finite difference analysis program to determine the amount of wattage required at the point of contact internally to produce the temperatures and pattern externally similar to that in the actual thermal image. Once the wattage has been established then apply the same analysis technique described in the
Figure 4. Wattage Approximation Example

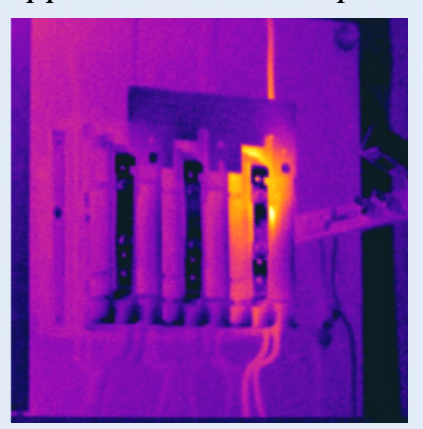

Data:

Fault temperature: $30^{\circ} \mathrm{C}$

Temp Rise above normal: $7^{\circ} \mathrm{C}$ Load: 6 Amps

Rated Capacity: 200Amps

Copper melting voltage: 0.43 Volts

Analysis

From the heat affected zone and average temperature, guess at the amount of heat dissipation created by the fault. Likely 2-3 Watts but make a very conservative assumption of "at least"1 Watt

Fault power: $P=I^{2} R$ or

Fault resistance $R=P / I^{2}$

In this case $=1 / 6 * 6=0.028$ ohms. or 28 milliOhms

(a typical value $<10$ micro-Ohms)

\section{Assessment}

What is the voltage drop across the connection and how close is it to the melting voltage?

Voltage drop at the connection: deltaV $=I * R=0.17$ volts or about $\underline{40 \%}$ of the melting voltage!

At what current value will the melting voltage be achieved? deltaV/R $=I=0.43 / 0.028=15$ Amps At about 15amps the melting voltage will have been achieved. This will generate about 7 watts of heat so the failure may be classified as functional but not necessarily catastrophic. At 100 Amps (50\% load) however275 Watts of heat will be generated and a catastrophic failure likely.

Conclusion: Very high probability of failure if the load increases. 
wattage approximation method to establish proximity to melting voltage/temperature under the different design currents. The advantage of this technique is that wattage determination will be more precise and, if desired the transient situations associated with design criteria (2) and (3) can also be analyzed.
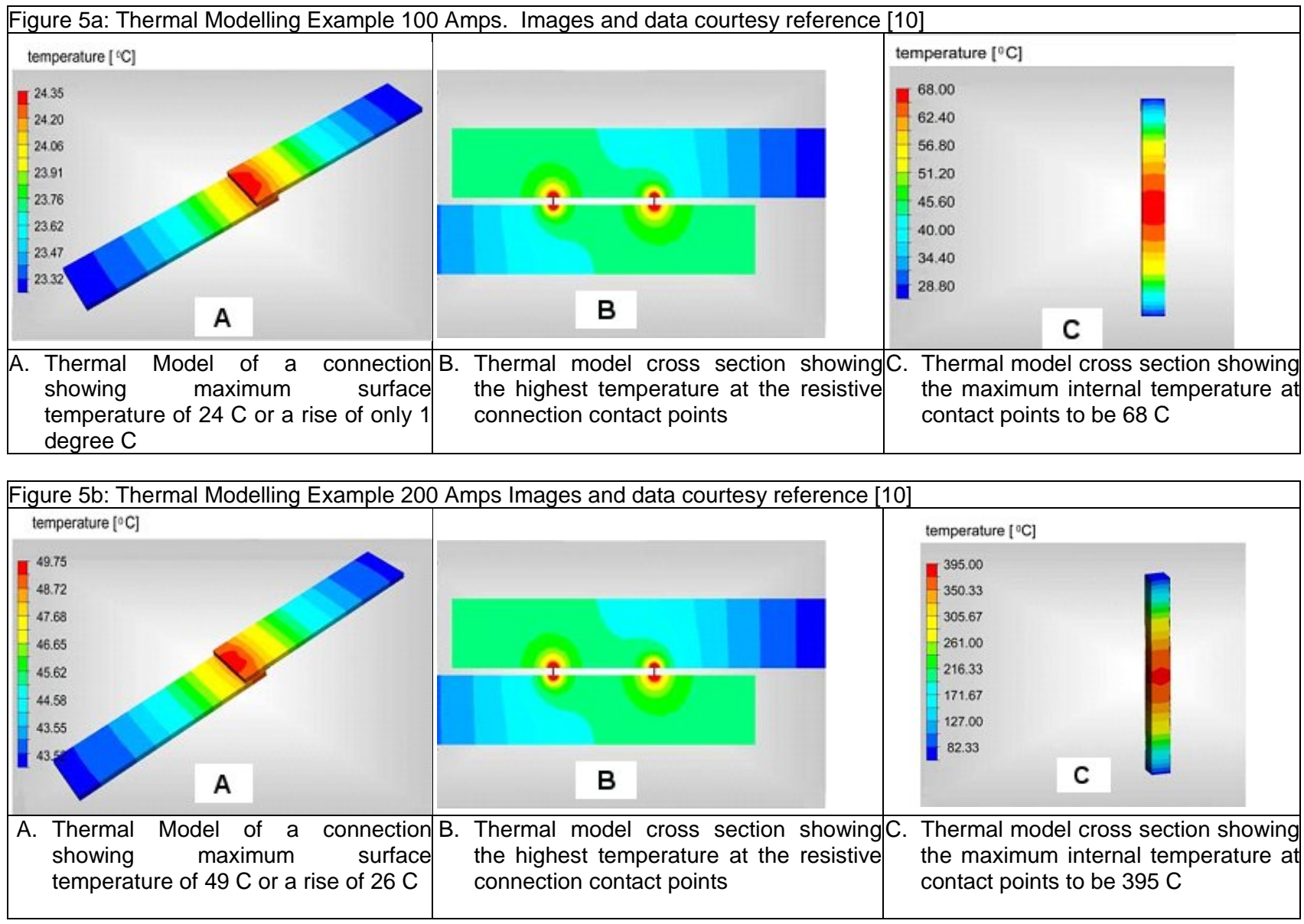

\subsection{Monitoring as a means of failure assessment}

What if thermal modelling or the wattage approximation methods cannot be utilized? (such as the case when load was not measured) When these methods are possible or practical, then the only other solution left to minimize risk is that of monitoring on either a continuous or interval basis.

\subsubsection{Continuous monitoring}

Of the two type of monitoring, continuous monitoring of a connection thermal anomaly is the preferred (but more costly) method. In this method an infrared camera, (sometimes it may be possible to use a spot radiometer) is set up to continuously observe the connection with a rate of rise and threshold temperature alarm sent to a remote monitoring station or control room. This type of monitoring is really only effective when direct line of sight to the connector is possible, such as out-door substations, and in switchgear cabinets which have infrared windows installed. Typically, however line of sight to the connection is only achieved when a cabinet door is open or panel cover removed, and this must be the case as well for continuous monitoring creating a potentially un-safe work condition. As infrared cameras and spot radiometers are getting quite small, it may be feasible to mount them with adequate clearance inside the cabinet with the door or panel closed. Continuous monitoring has the distinct advantage of being able to potentially deal with an unexpected stressor triggering a catastrophic connection failure. It has the disadvantage of the capital and setup costs associated with procuring and temporarily installing the monitoring and support equipment. Large facilities should consider procuring such a system in advance, and have them ready to deploy in the event an anomaly on a critical component is detected. As infrared cameras continue to fall in price, simultaneous with increase in performance and network interfacing capabilities this approach becomes both feasible and cost effective in for thermal anomalies detected in critical equipment and situations where shutdown for connection repair is not feasible or extremely costly. 


\subsubsection{Interval monitoring}

While less preferable than continuous monitoring, interval monitoring may be the only feasible method for trying to minimize the risk catastrophic. Interval monitoring simply consists of inspecting the "potential" failure at a high enough frequency to identify when a functional failure is imminent. This is typically done by establishing both rate of rise and absolute alarm limits. The problem with interval monitoring is that is assumes the failure will be one of component degradation, typically in response to a stressor (see below) but this type of monitoring will be ineffective against the unexpected event stressor such as a short circuit or surge. Management must be prepared to accept that while the likelihood of this unexpected event may be low, the chance of catastrophic failure is real, and the consequence in such event should be acceptable.

Once an anomaly has been found and interval monitoring is the chosen solution then the interval should be the highest available for the given resources. If there are known stressors on the component then the interval should be higher than if there are not. If there are time-dependent stressors (such as peak load, chlorine wash-down, or construction activity) then monitoring should take place as close in time as possible after the stressor occurs.

\subsubsection{Frequency of inspection}

Nolan and Heap developed the concept of a "P-F" failure curve shown in Figure 4. While there are many variations of this concept the premise is all the same. The "P" point is the point of potential failure detection (in this case by thermography) which is followed by a failure development period until complete failure " $F$ " occurs. They suggest that the proper predictive maintenance interval be defined by the time between detecting a potential failure to the time of a functional failure divided by two. This is the classic $(\mathrm{P}-\mathrm{F}) / 2$ formula shown in Figure 4 with various failure stressors. The Failure point " $F$ " represents failure due to degradation and functional failure to meet design criteria 1,2 or 4 . The dotted lines represent a design criteria 3 failure: an unpredictable occurrence of an instantaneous extremely high fault current.

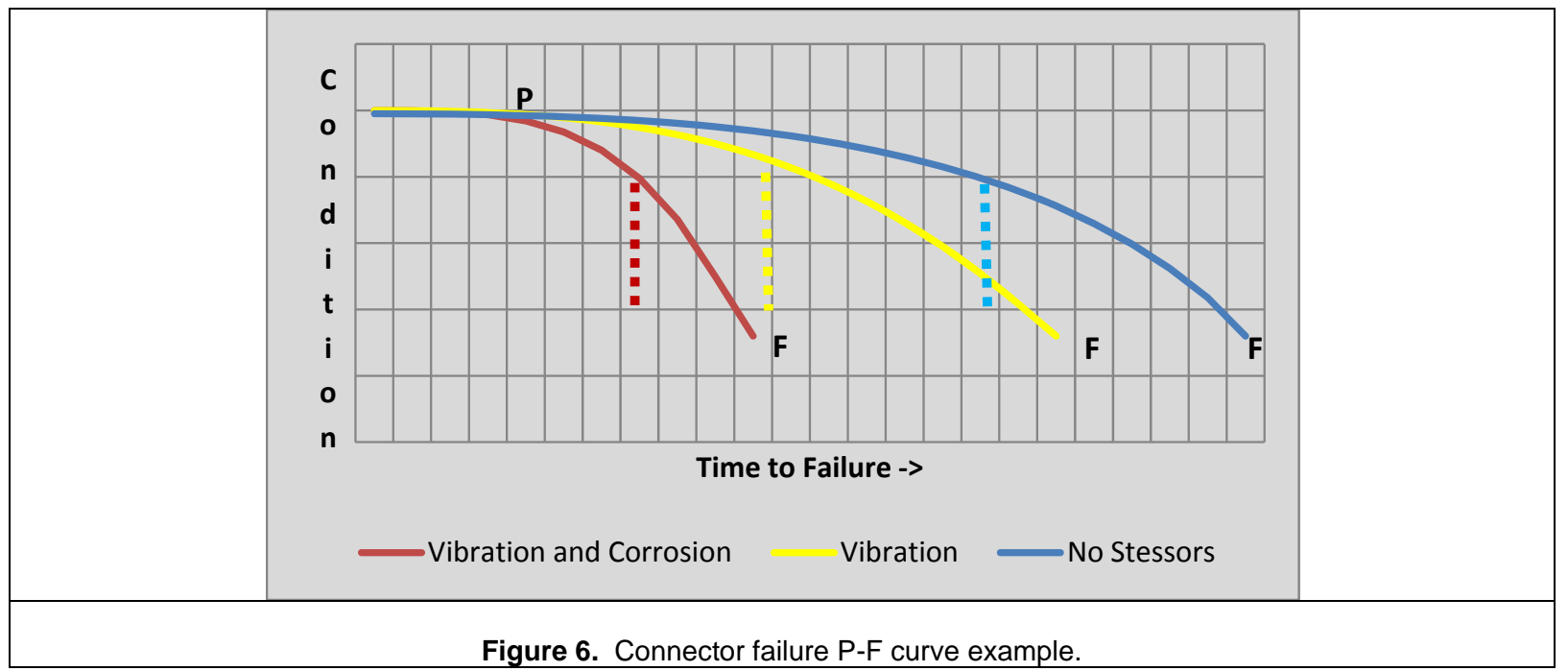

\subsubsection{Failure Stressors and Situations}

The following are some examples to illustrate why the P-F curve will be different given different situations.

Situation 1. A normal maximum current is flowing in the circuit every day for 24/7. The room air temperature is controlled and contains no adverse corrosive agents nor external vibration. Failure mechanisms in a faulty connector are minimized.

Situation 2. Exactly the same as situation 1 but the circuit is turned off and on frequently every day. A failure mechanism exists due fretting corrosion created by thermal expansion and contraction as the thermal anomaly in the connector heats up and cools down each time the circuit is turned on and off respectively. 
Situation 3. The same as situation 1 but the connector thermal anomaly is located in a water treatment facility, public swimming pool, food processing plant, barn, pulp and paper mill, or chemical plant where corrosive environments such as chlorine, ammonia or sulphates are present. A failure mechanism exists due to the presence of a corrosive element.

Situation 4. The same as situation 1 but the thermal anomaly is located in an environment with externally induced vibration. While some connector designs expect this ( e.g.: a motor junction box connector) other sources of vibration are not always anticipated by the electrical design engineer (the proximity of a reciprocating compressor, construction, truck traffic, etc.)

When multiple stressors exist simultaneously the time to failure can be quite rapid. A thermal anomaly in a connection in a $50 \mathrm{Hp}$ motor control unit in a nuclear power plant may have a p-f time of 3 to 4 years resulting in a time to inspection of 2 years (which is the EPRI re-commended maximum inspection time interval) while in a meat packing operation or water treatment plant with all 4 stressors working in combination the p-f time can be as low as 6 months requiring a consequent inspection interval of every 3 months.

\section{Electrical Connection Attached to a Thermal Device}

Evaluating electrical connections attached to thermal devices such as fuses, circuit breakers, contactors, transformers or capacitors can be tricky since, in abeyance with the second law heat must flow from the hot to cold, and prefers the path of least resistance which is typically the cooler and highly conductive (both thermally and electrically) wires. In order to get to the wire heat will have to pass through the connector hence making it warmer than the wire (not in this case to a "functional fault" condition. Here we must rely on component, pattern, temperature, and situation to evaluate whether there is in fact a problem with the connection. If the thermal device is exposed with a good line of sight the analysis is simple and straightforward: Which is warmer: the component or the connection?. If the component is warmest then the connection does not have a problem. If the connection is warmest then the connection has functionally failed. This in fact is a more serious situation that a simple connection between two unheated conductors since the connection may force the thermal device to run warmer than normal and "functionally fail". An example of this would be a fuse or overload. When this occurs we sometimes observe a thermal pattern of heat flowing out of the opposite side of the thermal device as this becomes the thermal path of least resistance. In three phase systems we can also use the principle of phase to phase pattern comparison, and in single phase systems we can compare the pattern on the line side to the load side of the device.

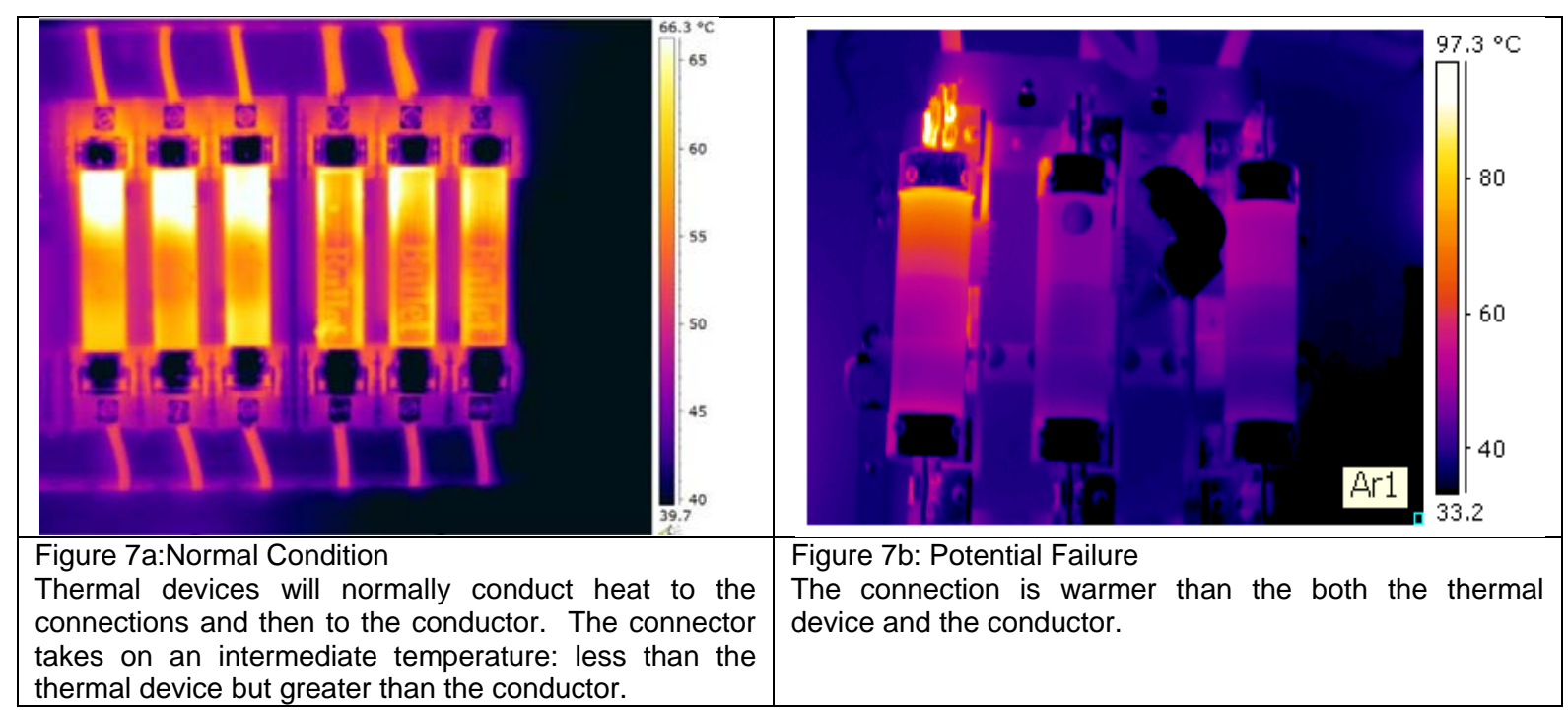




\section{Summary}

Failure prediction of an electrical connection based upon temperature alone is not accurate, and at times can be very misleading. Until we know about the problem root cause; all the materials involved with their thermal and other material limits; the extremes of load variance; and the unusual environmental and operational stressors that will be placed on the component then determining whether a thermal anomaly is a potential or functional failure will be extremely difficult

The solutions to the dilemma about what to do about a connector thermal anomaly are (in order of preference):

1) If any known material thermal limit is exceeded then assume a functional failure has occurred and repair it. In most cases for a connection this will be the lesser of $90^{\circ} \mathrm{C}$ or the wire insulation limit.

2) If (1) has not been established then assume all connection thermal anomalies are functional failures and schedule them for repair.

3) If (2) is not possible and you do not know whether a problem is a functional or potential failure then perform a wattage or modelling analysis to establish the approximate micro-ohm value of the connection fault. Then using that value and the 3 design load criteria establish whether melting voltage will occur at any of these values. If it will, then functional, and perhaps complete or catastrophic failure is likely.

4) If (3) is not possible or reliable, then consider utilizing continuous or interval monitoring. Recognize, however, that if an unusual stressor happens that this method may not be reliable to prevent complete or catastrophic failure. If an unusual stressor occurs then check the connector as soon as possible after it has occurred to see whether temperature has increased.

Failure prediction of electrical connections should not be performed based upon temperature alone but when necessary should be based upon proper risk analysis techniques. The reality of human nature is not to remember the hundreds of times that thermography identified a problem and it was repaired before failure, but rather the one catastrophic failure that occurred because it was misdiagnosed as a "minor anomaly".

\section{Acknowledgements}

The author would like to acknowledge the technical information, insights and editing provided by Dr. Roland Timsit of Timron Scientific Consulting Inc.. Dr. Timsit has a wealth of knowledge and experience in electrical contact and connectors, and shares considerable information on his website. Many related papers, including some of those referenced here may be downloaded for free from http://www.timron-inc.com/

The author also acknowledges the on-going work and concepts on electrical severity assessment contributed by all the team members of The Snell Group, but in particular the editing and insights provided by Roy Huff. Analysis of thermal imagery is an ongoing and evolving field and The Snell Group provides significant up-to date information and downloads on infrared thermography on its website www.thesnellgroup.com and message board www.IRTalk.com.

\section{REFERENCES}

[1] NFPA 70B, Recommended Practice for Electrical Equipment Maintenance, National Fire Protection Association, 2006

[2] McIntosh, G.B., NFPA 70B Fault Severity Guidelines for Electrical Thermography, Ontario Electrical Contractors Magazine, 2010

[3] UL standard 489. Underwriters Laboratories, Molded-Case Switches and Circuit-Breaker Enclosures

[4] Pauley, J, Square D Company, Bulletin No. 0110DB9901R2/02, Wire Temperature Ratings and Terminations, March 2002

[5] Nowlan, F.S, Heap, H.F., Reliability Centered Maintenance, Report A066-579, 1978

[6] Moubray, John, Reliability-Centred Maintenance RCM II, Elsevier Sci \& Tech Book Div; 2nd edition, 1999

[7] ISO 14224, International Standards Organization, Petroleum, petrochemical and natural gas industries -Collection and exchange of reliability and maintenance data for equipment, 2006

[8] Dodson, Nolan, Reliability Engineering Handbook, CRC Press; 1999

[9] Timsit, Roland, S. The Connector TekNote, May 2001, Timron Scientific Consulting Inc., www.timroninc.com

[10] Timsit, R.S., Wilson, C., McIntosh G., Contact Spot Temperature and the Temperature of External Surfaces in an Electrical Connection, Proceedings of ICEC\&ICREPEC, 2012 